\title{
ESTIMATING HOUSING VACANCY RATE IN QINGDAO CITY WITH NPP-VIIRS NIGHTTIME LIGHT AND GEOGRAPHICAL NATIONAL CONDITIONS MONITORING DATA
}

\author{
Xiao Niu ${ }^{1, *}$ \\ ${ }^{1}$ Shandong Provincial Institute of Land Surveying and Mapping - xiaoniu710@126.com
}

Commission III, Urban Sensing and Mobility

KEY WORDS: Housing vacancy rate (HVR), NPP-VIIRS, Nighttime light data, Geographic National Conditions Monitoring Data (GNCMD), resident population distribution data, SVM, Moran's I

\begin{abstract}
:
Accompanying China's rapid urbanization in recent decades, especially in the new millennium, the housing problem has become one of the most important issues. The estimation and analysis of housing vacancy rate (HVR) can assist decision-making in solving this puzzle. It is particularly significant to government departments. This paper proposed a practical model for estimating the HVR in Qingdao city using NPP-VIIRS nighttime light composed data, Geographic National Conditions Monitoring data (GNCMD) and resident population distribution data. The main steps are: Firstly, pre-process the data, and finally forming a series of data sets with 500*500 grid as the basic unit; Secondly, select 400 grids of different types within the city as sample grids for SVM training, and establish a reasonable HVR model; Thirdly, using the model to estimate HVR in Qingdao and employing spatial statistical analysis methods to reveal the spatial differentiation pattern of HVR in this city; Finally test the accuracy of the model with two different methods. The results conclude that HVR in the southeastern coastal area of Qingdao city is relatively low and the low-low clusters distributed in patches. Simultaneously, in other regions it shows the tendency of the low value accumulation in the downtown area and the increasing trend towards the outer suburbs. Meanwhile the suburban and scenery regions by the side of the sea and mountains are likely to be the most vacant part of the city.
\end{abstract}

\section{INTRODUCTION}

The housing vacancy rate (HVR) is a significant indicator to analyze and assess the health condition of the real estate market which directly affect the life quality of local residents (Chen et al. 2015). Since the reform and opening up, especially in the new millennium, China has been experiencing urbanization at a rapid speed ( $\mathrm{Li}$ et al., 2017; Wu 2015). Nevertheless, the housing problem has become one of the most important issues for the government and residents. Although the per capita residential area is continuously increasing, there still exists the problem of imbalanced supply structure and insufficient effective demand (Yang and Sun 2004). In some cities or districts, people are suffering from the shortage of legal residence and the high rising real estate prices. Meanwhile, the empty city or ghost city phenomenon (Powell 2010) has been reported occasionally, for the low occupancy rate and high HVR in some other ones. For the municipal and planning authorities, obtaining the housing vacancy rate and its spatial distribution is one of the crucial ways to analyze and solve this imbalance of structure of urban housing. Due to the variability and randomness of the population, investigating HVR by field survey requires lots of human and economic resources. Therefore, it is the most economical and time-saving way to estimate HVR using remote sensing techniques.

Nighttime light data are typical remote sensing data which can indicate population activities. Therefore, the data have been used to study in multiple fields such as economic development (Forbes 2013, Tang, Cheng and Qu 2013), urbanization (Liu et al. 2012, Zhang and Seto 2013, Zhou et al. 2014), population density (Sutton et al. 1997, Zhuo et al. 2009), resource consumption (Cao et al. 2014, Xie and Weng 2016), environmental monitoring (Huang et al. 2015, Ou et al. 2015, Zhang et al. 2017) and other human activities. Time series data from the Defense Meteorological Satellite Program's Operational Linescan System (DMSP-OLS) archived by the National Oceanic and Atmospheric Administration's National Geophysical Data Center (NOAA/NGDC) of the United Sates are the most commonly used type of the nocturnal light data (Elvidge et al. 1997). And the annual average, stable and could free composed data can download from 1992 to 2013 in the website (https://ngdc.noaa.gov/eog/dmsp/downloadV4composites.html). The visible pixel are relative values ranging from 0 to 63 rather than absolute values in Watts per $\mathrm{m}^{2}$. Therefore, DMSP_OLS data have some shortcomings such as the lack of on-board calibration (Liu et al., 2012) and the saturation in the bright cores of urban centers (Letu et al. 2012, Ma et al. 2014). The Suomi National Polar-orbiting Partnership satellite carrying the first Visible Infrared Imaging Radiometer Suite (NPP-VIIRS) instrument was launched On October 28, 2011 (Elvidge et al. 2013, Miller et al. 2012). Since then, the Earth Observation Group at NOAA/NGDC generate monthly average data from April 2012 to February 2018 and annual average data in 2015. The VIIRS Day/Night Band (DNB) collects earth's surface lowlight imaging data which provides several key improvements over DMSP-OLS data, including wider dynamic range, in-flight calibration, higher spatial resolution etc. (Elvidge et al. 2017, Miller et al. 2013). In several recent studies, it has been shown that NPP-VIIRS nighttime light data has a stronger capacity than DMSP-OLS data in estimating socioeconomic phenomena. For instance, Jing et al. (2015); Li et al. (2013); Shi et al. (2014a) revealed the availability of NPP-VIIRS for modelling socioeconomic indicators. Shi et al. (2014b) demonstrated than NPP-VIIRS data have higher spatial accuracies in extracting the built-up urban areas than DMSP-OLS data at national or regional scale. Additionally, Ou et al. (2015) compared the stable light and calibrated products from DMSP-OLS with the new-generation 
nighttime light data from NPP-VIIRS for studying the spatial distributions of global fossil fuel carbon dioxide emissions. And the result showed that NPP-VIIRS data are proper for the emission map.

In some developed countries and regions include Hong Kong ${ }^{1}$ and Tai wan (Hsueh, Tseng and Hsieh 2007) of China, the statistical work of HVR have already accumulated a set of detailed statistics system. However, there is no authoritative organization or department in the country to carry out this work. At present, many scholars have used existing data to study HVR. For example, Yao and Li (2011) compared brightness of night light with house price, and provided a new way to get house vacancy information. Zhu and Yan (2011) established a reasonable residential HVR model using house price change rate as a dependent variable. And they worked much on the vacancy characteristics and HVR in Hangzhou through the field survey and GIS analysis. While these researches can get useful information, they don't directly provide the spatial distribution of HVR. Chen et al. (2015) proposed a method for estimating the HVR in metropolitan areas using NPP-VIIRS nighttime light composite data and visualized the HVR on maps. Zheng et al. (2017) developed a ghost city index (named GCI), which comprising three criteria: consistency of lit area and built-up area, illumination intensity and population density, to evaluate the intensity of ghost city phenomenon in Yangtze River Delta. However, these studies mainly focus on establishing a simple linear model between the intensity of lighting radiance and urbanization ratio or residential building density without considering the influence of the type and height of building area. In this paper, we presented a more accurate model for estimating the HVR in Qingdao city using NPP-VIIRS DNB, building area of Geographic National Conditions Monitoring data (GNCMD).

\section{STUDY AREA AND DATA USED}

\subsection{Study area}

Qingdao, a representative city of Shandong Province, is selected as the research object. Qingdao has a population of 9.2 million people. The per capita GDP of this city is relatively high comparing with most cities in China, but varies a lot in different districts, from lower than 10000 USD to nearly 30000 USD. This variety indicates the imbalance of housing in this city. Studies on related subjects, including vacancy rate, should also be representative and valuable.

The population and GDP of Qingdao is shown in Table 1. GDP of the Free Trade Port Area is merged into the Huangdao District where it lies in. To make the data more comparable with other cities in the world, GDP in Chinese Yuan (CNY) is converted into GDP in US Dollar (USD) using the average exchange rate in $2016(6.64: 1)$.

\begin{tabular}{|l|r|r|r|}
\hline \multirow{2}{*}{ Region } & \multirow{2}{*}{$\begin{array}{c}\text { Population } \\
\text { (thousand) }\end{array}$} & \multicolumn{2}{|c|}{ GDP (thousand USD) } \\
\cline { 3 - 4 } & total & per capita \\
\hline Whole Municipality & $\mathbf{9 2 0 4 . 0}$ & $\mathbf{1 5 2 0 3 9}$ & $\mathbf{1 6 . 5 2}$ \\
\hline Shinan District & 580.3 & 15307 & 26.38 \\
Shibei District & 1083.7 & 10399 & 9.60 \\
Licang District & 553.3 & 5362 & 9.69 \\
Laoshan District & 435.5 & 8285 & 19.03 \\
Huangdao District & 1515.9 & 43239 & 28.52 \\
Chengyang District & 702.3 & 14053 & 20.01 \\
Jimo & 1214.5 & 17778 & 14.64 \\
Jiaozhou & 885.2 & 15601 & 17.62 \\
Pingdu & 1372.6 & 12240 & 8.92 \\
Laixi & 761.0 & 8439 & 11.09 \\
Development Zone & 99.7 & 1336 & 13.40 \\
\hline
\end{tabular}

Table 1. Population and economy of Qingdao (Qingdao Statistics Bureau, 2017)

\subsection{Geographic National Conditions Monitoring data}

Geographic National Conditions Monitoring has been a key project organized by the First National Geographic Census Leading Group Office of the State Council of China for years. In this project, Global Navigation Satellite System (GNSS), remote sensing(RS), geographic information system (GIS) and other modern surveying and mapping technology are integrated, and historical archives are utilized, to monitor and analyse the status, distribution and changes of natural and cultural geographical features in China. Since 2015, three GNCMD productions (i.e., GNCMD 2015, GNCMD 2016, GNCMD 2017) have been released. And the GNCMD 2017 which has a new classification hierarchies (GQJC 03-2017) include 10 Level I classes (as shown in Figure 1), 59 Level II classes, and 143 Level III classes, is the latest data. Meanwhile, the precision of this production is very high. To estimate HVR in a fine grain, residential quarters and building information data (detailed classification information shown in Figure 1) are required from GNCMD 2017. residential quarters are stored as point features with multilevel categories and other attributes; building blocks are stored as polygon features with a category of the top level.

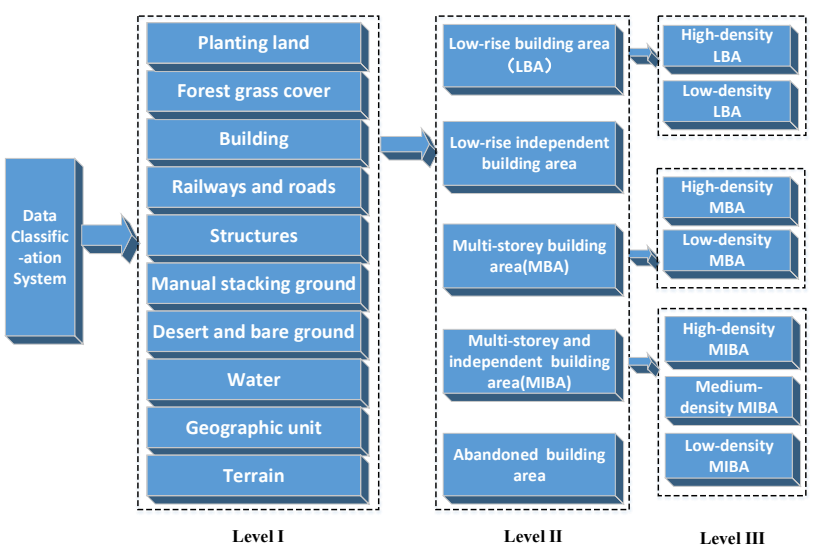

Figure 1. Geographical National Conditions Monitoring Data classification system (only including all Level I classes, Level II building classes and Level III building classes)

\footnotetext{
${ }^{1} 2011$ Hong Kong property Review
} 


\subsection{NPP-VIIRS nighttime light data}

In this study, we obtained the available monthly average composite NPP-VIIRS nighttime light data with a spatial resolution of 15 arc-seconds (about $500 \mathrm{~m}$ ) from the website of NOAA/NGDC (https://ngdc.noaa.gov/eog/viirs/download_dnb _composites.html). To integrate with the GNCMD, imageries are projected into the same spatial reference (the Gauss-Kruger projection), then resampled to $500 \mathrm{~m} * 500 \mathrm{~m}$ cell size to get the same spatial resolution. After correcting and clipping with the administrative boundary, the nighttime light data of Qingdao is extracted.

According to the instructions of this dataset, the primary version series of monthly composites has not been filtered to screen out lights from aurora, fires, boats, and other temporal lights while the annual composites have filtered layers. To assure the accuracy, we examined the quality of monthly average imagery in 2017 by comparing with the most recent version of annual products and a map of Qingdao. The result shows that the image of October 2017 is the best choice for this study, there's scarcely any unexplainable outlier value. However, there exists background noise in monthly products. About $40 \%$ of the pixel values in the annual image are larger than 0 , this ratio is applied in noise removal. $40 \%$ pixel values in October's image are larger than 1.04, so the image is filtered and shifted with this threshold. The result is shown in Figure 2.

Although the nighttime light data are proved to be enough for population estimation in macroscale (at provincial, municipal or district level), for microscale (e.g. in a 500*500 meter grid) usages the accuracy is less evidential. To get reliable population at this scale, mobile operator data are employed.

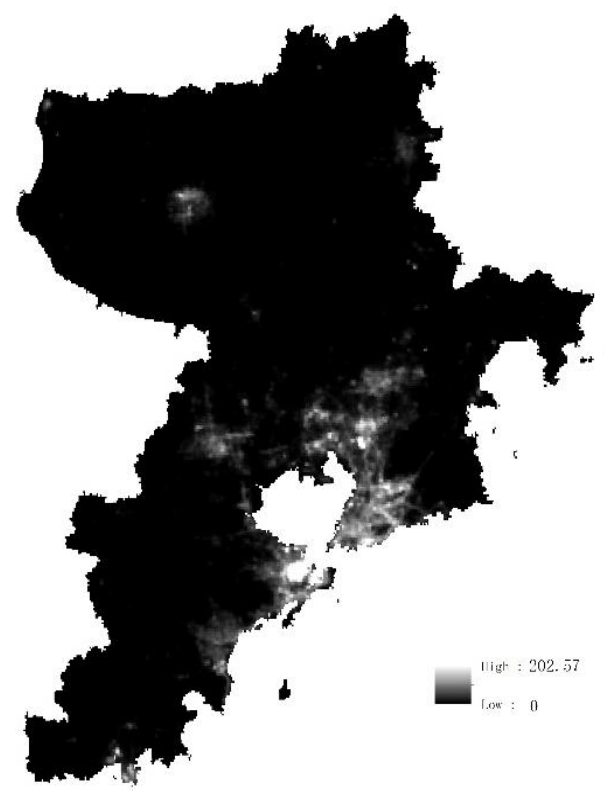

Figure 2. NPP_VRIIS nighttime light data in Qingdao city

\subsection{Population data}

In this paper, the residential population data from China Telecom one of the largest mobile operators in China will be introduced into our method. The monthly trajectories of users are analyzed and summarized to get this dataset. China Telecom has totally 255 million mobiles users in China, 1.3 million of them live in Qingdao. The amount of samples is huge and the sample ratio is high (more than 10\%) either in the state scale or city scale, this assured the quality of this dataset. The main disadvantage of this dataset is the expense, so it is used just for creating the training set for our HVR model; the estimation of the city is based on other datasets.

\section{METHODS}

\subsection{Analysis unit and sampling}

As mentioned above, datasets used in this research have different spatial resolutions. Therefore, the analysis unit should be selected before data pre-processing. A net of rectangular grids is created according to the administrative boundary of Qingdao. The grids are the same as GNCMD used, both the width and height are 500 meters. These grids should be filtered before use, because only residential area should be take into consideration. The residential area in the municipal districts of this city is $133 \mathrm{~km}^{2}$ (or $1.2 \%$ of the whole administrative area) by the end of year 2015; in the whole municipality is about double of the municipal districts (DRCnet 2018). It is surely that most of the grids can be ignored. To extract residential grids, GNCMD is introduced for spatial join. Finally, there are 2557 grids selected, the total area is 255 $\mathrm{km}^{2}$, consistents with statistics.

To create and validate the regression model, 500 sample are chosen from residential grids according to POIs, high-resolution remote sensing images and building data. These samples cover different parts of the city and different building types. IDs and boundaries of these grids are submitted to China Telecom to query the residential population.

\subsection{Variable selection}

To build a comprehensive and effective regression HVR model, several explanatory variables are carefully selected.

The first one is the nighttime light intensity $\left(l_{\text {light }}\right)$. HVR is determined by the number of house units and the resident population. Nighttime light are related to both houses and people, so their intensity is a major variable in the vacancy rate model.

Building data can be used for inferring the number of house units. GNCMD gives the detailed classification of building, including the height, density and area (As shown in Figure 1). In addition, the data of residential building area can be obtained according to residential quarters from the GNCMD. Based on this dataset, 4 variables, $r_{\text {all }}, r_{\text {main }}, h_{\text {main }}$ and $r_{\text {building }}$, are generated and applied in the model as explanatory variables. Given a grid, and the set of residential patches $\boldsymbol{P}$ in it,

$$
\begin{gathered}
r_{\text {all }}=\frac{\sum_{p \in \boldsymbol{P}} \operatorname{area}(p)}{\operatorname{area}(\text { grid })} \\
r_{\text {main }}=\frac{\sum_{p \in \boldsymbol{P}_{\text {main }} \operatorname{area}(p)}}{\operatorname{area}_{\text {marid })}}= \begin{cases}N_{h}, & \text { Type }_{\text {main }} \text { is MBA } \\
N_{l}, & \text { Type }_{\text {main }} \text { is LBA }\end{cases} \\
r_{\text {building }}=\frac{\sum_{p \in \boldsymbol{P}} \text { floor }_{\text {area }(p)}}{\text { area }(\text { grid })}
\end{gathered}
$$

where Type main $_{\text {in }}$ is the majority class of the patches

$\boldsymbol{P}_{\text {main }}$ is the patches whose class is Type $e_{\text {main }}$

$N_{l}$ is the average floor of LBAs

$N_{h}$ is the average floor of MBAs

floor_area $(p)$ is the floor area of buildings in patch $p$. 
These variables descripts the area and height of patches and buildings. Though we can calculate the quantity of house unit just with $h_{\text {main }}$ and $r_{\text {building }}, r_{\text {all }}$ and $r_{\text {main }}$ are introduced into the model to cooperate with nighttime light data.

The resident population data from China Telecom $\left(\right.$ pop $\left._{\text {res }}\right)$ is only used for calculating HVR of the samples and not directly applied in the model.

\subsection{HVR of sample calculation}

For a grid, the number of households $\left(n_{\text {res }}\right)$ is inferred from residential population. The average number of households in term of (Qingdao Statistics Bureau, 2017) is 3. Therefore, the formula is as follows.

$$
n_{\text {res }}=\frac{\text { respop }}{3}
$$

Then, we use GNCMD to deduce the quantity of house units.

$$
\begin{gathered}
a_{\text {res }}=250000 * r_{\text {building }} * h_{\text {main }} \\
n_{\text {house }}=\frac{a_{\text {res }}}{30}
\end{gathered}
$$

The formula (6) calculate the total area of the residence. And 250000 means the area of grid. According to (Qingdao Statistics Bureau, 2017), per capita living area is 30 square meters, so the number of house units can be gained in formula (7).

Based on the above variables, HVR can be acquired as following formula.

$$
\text { hvr }=\frac{n_{\text {house }}-n_{\text {res }}}{n_{\text {house }}}
$$

\subsection{SVM model}

The regression model is create using SVM, a popular machine learning method. SVM can cope with both linear regression problems and non-linear ones, and more adaptive with multidimensional data. Building a model using SVM means training it with sample data. 400 tuples are inputted into SVM. To get a better fitting, several types of kernel functions are tested, such as liner, polynomial, sigmoid and Gaussian. The kernel and corresponding model which can get the best fitting will be selected.

The SVM is trained using 400 sample grids which contain nighttime light intensity value, building parameters and HVR< $l_{\text {light }}, r_{\text {all }}, r_{\text {main }}, r_{\text {building }}, h_{\text {main }}, h v r>$ to obtain a regression model for the estimated HVR by these parameters. In contrast, the same data is used to establish a linear regression model.

\subsection{Model validation and result estimation}

Given parameters $<l_{\text {light }}, r_{\text {all }}, r_{\text {main }}, r_{\text {building }}, h_{\text {main }}, h v r>$ of some grids, their HVR can be estimated with the regression model. Before processing all the residential grids, the regression model should be validated first.

Calculate the goodness of fit, then compare the HVR of the 100 grids preserved before with their estimates to quantify the accuracy of the model. A linear regression model can be created as a reference. Then superimpose aerial imagery to check the area with the highest HVR to confirm the correctness of the model.
Finally, the HVR of all grids are estimated, then the spatial distribution and autocorrelation patterns of them are visualized and analyzed with the help of GIS software.

\section{RESULT AND DISCUSSION}

\subsection{Model accuracy evaluation}

(1) Goodness of Fit

Using the HVR model to compare the sample values and the estimates. The result is that $\mathrm{R}$ is equal to 0.436 and mean absolute deviation is 0.316 .

\begin{tabular}{|l|r|}
\hline Evaluation measure & Values \\
\hline Minimum deviation & -0.942 \\
Maximum deviation & 0.856 \\
Average deviation & 0.033 \\
Mean absolute deviation & 0.316 \\
Standard deviation & 0.399 \\
Correlation coefficient & 0.436 \\
\hline
\end{tabular}

Table 2. Goodness of fit

(2) Compared with linear regression

The model is evaluated with 100 reserved samples. When using the SVM method, the value of $\mathrm{r}$ (Pearson's Correlation Coefficient) is 0.532 , and mean absolute deviation is 0.499 ; When using the linear regression, the value of $r$ is 0.444 , and mean absolute deviation is 0.517 .

\begin{tabular}{|l|r|r|}
\hline \multirow{2}{*}{ Evaluation measure } & \multicolumn{2}{|c|}{ Values } \\
\cline { 2 - 3 } & SVM & Linear regression \\
\hline Minimum deviation & -0.627 & -0.73 \\
Maximum deviation & 0.98 & 2.171 \\
Average deviation & 0.431 & 0.445 \\
Mean absolute deviation & 0.499 & 0.517 \\
Standard deviation & 0.358 & 0.395 \\
Correlation coefficient & 0.532 & 0.444 \\
\hline
\end{tabular}

Table 3. Comparison between SVM and linear regression

The result of SVM regression is slightly better than linear regression. The mean absolute deviation is still large. But thanks to GNCMD and mobile operator data, this time we can obtain fine-grained and relatively reliable quantative evaluation of the HVR model. Previously, building data and residential population data were estimated mainly through macroscopic statistics or remote sensing images. Their accuracy at fine-grained levels was not validly verified.

The deviation is determined by the input dataset, model and analysis granularity. In this experiment, the possible reasons of the large deviation include: residential population can't be estimated accurately using nightlight data (discussed in 4.3); spatial autocorrelation is not take into the model; and the scale effects. Larger the scale, smaller the deviation between aggregated observations and estimates; smaller the scale, larger the heterogeneity and randomness of the deviations.

In order to further make clear of the model's quality, we compared the estimated HVR with aerial images of Google Map. Grids are classified into 5 groups according to their HVR values using the natural breaks (Jenks) method, and rendered with gradient colors. Blue stands for low HVR and red stands for the opposite. 6 grids with the highest HVR are selected, shown in Figure 3(a). 


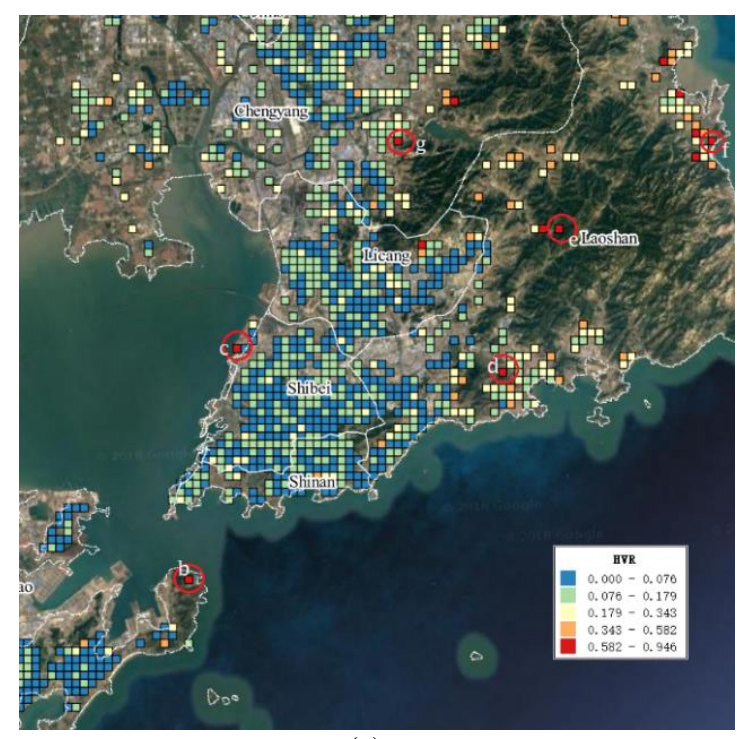

(a)

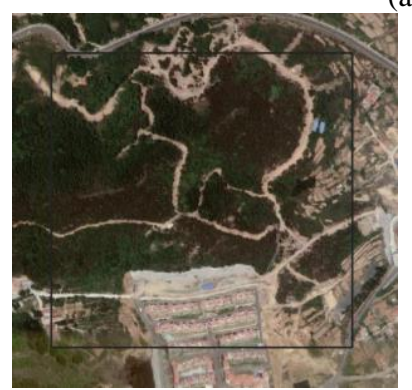

(b)

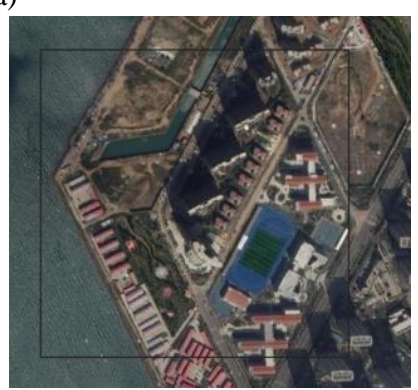

(c)

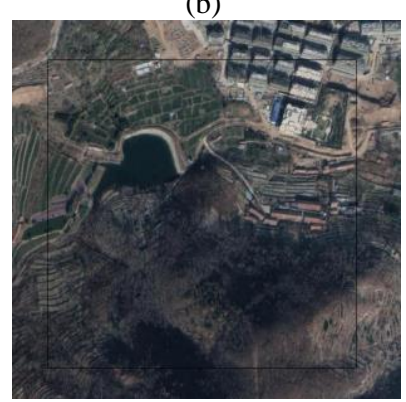

(d)

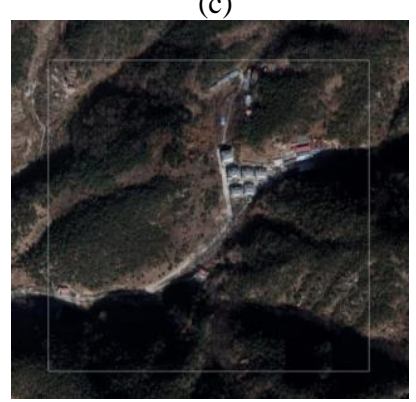

(e)

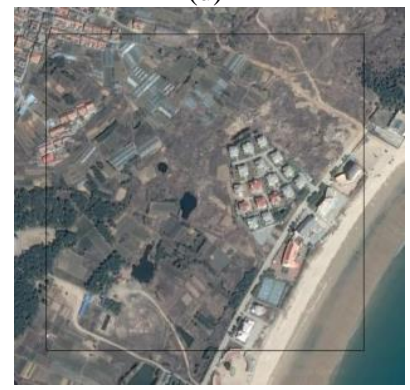

(f)

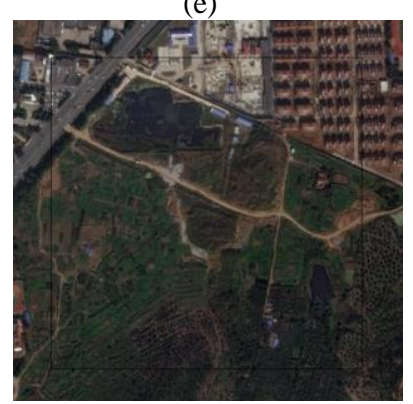

(g)

Figure 3. Comparison of the estimated HVR with Google Map in 2018. (a) shows the location of selected grids. (b), (c), (d), (e), (f) and $(\mathrm{g})$ are zoomed aerial images of selected grids in (a).

Buildings in Figure 3(b), (c), (d), (e) and (f) are adjacent to undeveloped (natural/rural) areas, bare ground or construction sites, so they are likely to have high vacancy rate. However, there's many regular buildings likely to be residential in (g). Is it really vacant? We overlaid this grid on Baidu Map in 2016 (Figure 4), and found out there's no such buildings. That means these buildings are newly constructed and are possibly not ready for use. After validated the HVR of these grids, we believe that though the mean absolute deviation is high, the most vacant (and most useful) part of the estimation result is reliable.

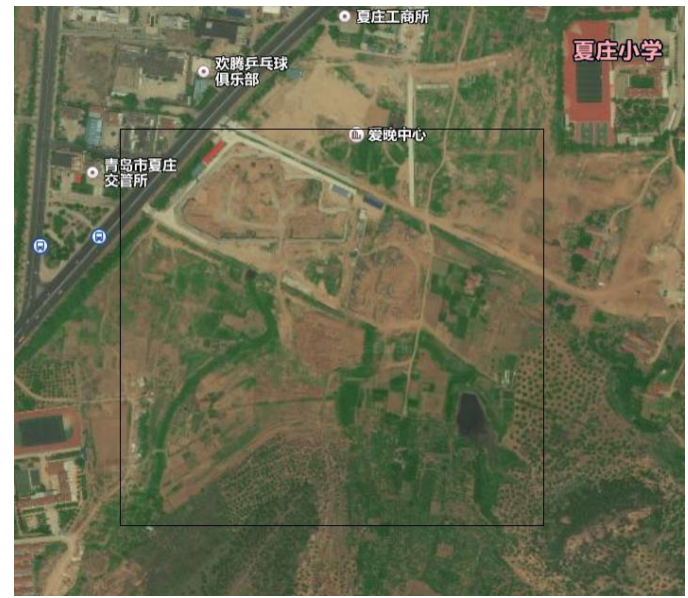

Figure 4. Grid (g) on Baidu Map in 2016

\subsection{Distribution and autocorrelation of HVR}

With the experimental result, a map showing the HVR of residential grids in Qingdao is created (Figure 5).

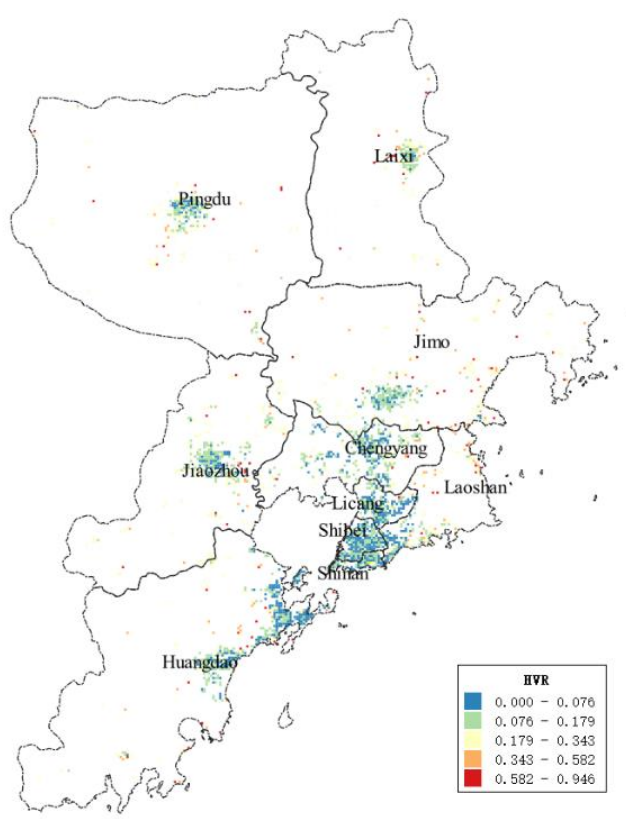

Figure 5. The spatial distribution of HVR in Qingdao

In Figure 5, it is clear that the downtown districts and county center areas in Qingdao city (blue grids in the map) have few vacant houses. As a whole, the overall vacancy rate in the southeastern coastal area is relatively low. Simultaneously, the concentration of housing is high and the housing density is relatively large. And HVR in other regions shows the tendency of the low value accumulation in the downtown area and the increasing trend towards the outer suburbs. Meanwhile the suburban and scenery regions (red and orange grids) by the side of the sea and mountains are likely to be the most vacant part of the city. Considering the relatively high price of real estates and the inconvenience in commute and living, it is reasonable that most people would prefer to live in other regions. 


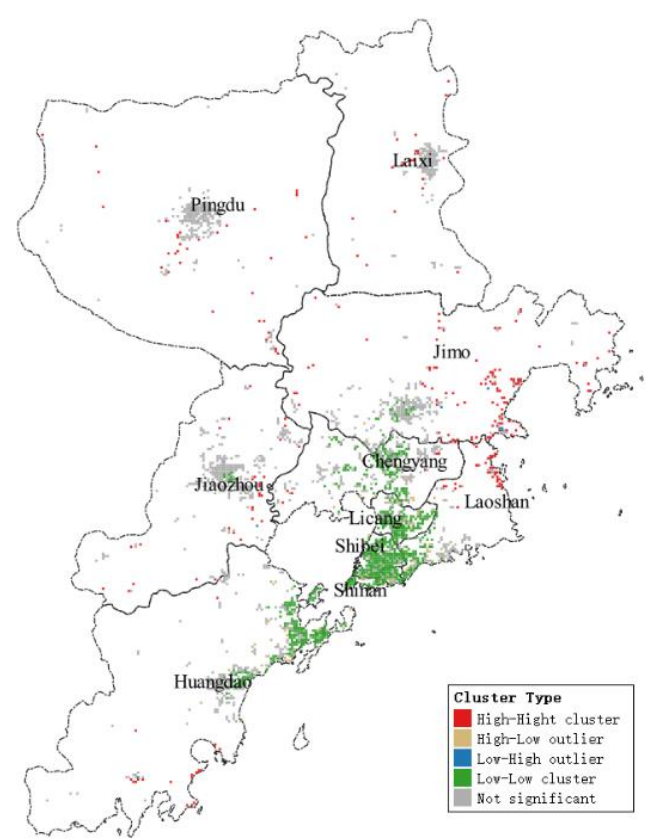

Figure 6. Cluster type of estimated HVR

Global Moran's I of HVR in Qingdao is calculated using ArcGIS's toolbox. The global Moran'I is 0.17 , the $\mathrm{p}$ value is less than 0.01 and the $\mathrm{Z}$ scores is 2.58 , indicating that HVR in Qingdao has significant autocorrelation. The grid with higher vacancy rate tends to cluster, and the grid with lower vacancy rate also tends to cluster. Consequently, the local Moran's I is calculated to locate the spatial autocorrelation patterns; the clusters are shown in Figure 6. The low-low and high-high clusters highly coincident with the downtown districts, county center areas and the suburban and scenery regions in Figure 5, validated the visual interpretation.

From the perspective of the spatial distribution of HVR on the county level units, the low-low clusters distributed in patches are relatively obvious. They are mainly distributed in Chengyang District, Shinan District, Shibei District, northeastern Huangdao District, southwestern Laoshan District and other coastal areas. The high-high values are mainly distributed in the eastern coast of Laoshan District and Jimo, and also the suburbs of other counties. The low-high and high-low values have no obvious clustering distribution, only scattered in the peripheral of highhigh and low-low regions.

This result demonstrate that the authorities should place stricter controls on vacant regions (seaside, hillside, etc.) to avoid the devalue of land, waste of resource, destruction of environment and real estate bubble. Meanwhile, more residential land should be supplied and more public facilities should be constructed in residential suburbs to improve both the living condition of residents and the efficiency of the whole city.

\subsection{Limitations of the model}

This research focuses on the HVR of cities and towns, our model only takes residential regions lie in urbanized areas into consideration. In rural areas, scale of settlements is relatively small; it is not easy to distinguish residential buildings from other buildings; and the nighttime light intensity tends to be very low due to the habits and customs of villagers. So, this method cannot directly apply to the estimation of HVR in rural areas.

\subsection{Extra analysis}

As a supplement of the discussion above, the correlation coefficient between residential population and the intensity of nighttime light is 0.34 in our experiment, indicates the relationship between them is not very strong. That means, in Qingdao, the fine-grained distribution of population cannot be fetched just using the nighttime light dataset; this conclusion is also likely to be valid in other Chinese cities. This is quite different from cities in developed countries. Construction sites throughout the city contributed a lot of nightlight, but most residents will come after months or years; transport facilities also give out more light than residential buildings. This is common in rapidly urbanizing countries. Therefore, it is reasonable to introduce extra variables, such as building height and area into the HVR model.

\section{CONCLUSION}

To get fine-grained quantative description of the housing vacancy phenomenon, one of the most concerned problem in China's urbanization progress, this paper presents an approach based on remote sensing methods. We integrated nighttime light, GNCM and mobile operator data together, established and validated a machine learning model of HVR in Qingdao. Following the suggested procedure and methods, administrative departments can get the HVR of the whole city in a short time at a reasonable cost. Though the accuracy of the experimental result is not high currently, it is still valuable for public policy decision-making.

In the future, it is important to make better use of GNCMD in the vacancy rate model. With more ground details, the nightlight data should be more applicable for finding, analysing and solving the imbalance problem of cities.

Besides, utilizing the spatial autocorrelation to improve the regression model and reduce its mean absolute deviation will be focus of our consequent research.

\section{REFERENCES}

Cao, X., Wang, J., Chen, J and Shi, F. 2014. Spatialization of electricity consum J.ption of China using saturation-corrected DMSP-OLS data. International Journal of Applied Earth Observations \& Geoinformation 28 (1), pp:193-200.

Chen, Z., Yu, B., Hu, Y., Huang, C., Shi, K and Wu, J. 2015. Estimating House Vacancy Rate in Metropolitan Areas Using NPP-VIIRS Nighttime Light Composite Data. IEEE Journal of Selected Topics in Applied Earth Observations \& Remote Sensing 8 (5), pp:2188-2197.

DRCnet 2018. DRCNET Statistical Database System. The Information Website of Development Research Center of the State Council. http://www.drcnet.com.cn (1 January 2018).

Elvidge, C., BAUGH, K., HOBSON, V., Kihn, E., Kroehl, H., Davis, E and Cocero, D. 1997. Satellite inventory of human settlements using nocturnal radiation emissions: a contribution for the global toolchest. Global Change Biology 3 (5), pp:387395.

Elvidge, C. D., Baugh, K., Zhizhin, M., Feng, C. H and Ghosh, T. 2017. VIIRS night-time lights. International Journal of Remote Sensing 38(21), pp:5860-5879. 
Elvidge, C. D., Baugh, K. E., Zhizhin, M and Hsu, F. C. 2013. Why VIIRS data are superior to DMSP for mapping nighttime lights. Proceedings of the Asia-Pacific Advanced Network 35, pp:62-69.

Forbes, D. J. 2013. Multi-scale analysis of the relationship between economic statistics and DMSP-OLS night light images. Mapping Sciences \& Remote Sensing 50 (5), pp:483-499.

Hsueh, L. M., Tseng, H. P and Hsieh, C. C. 2007. Relationship Between the Housing Vacancy Rate, Housing Price, and the Moving Rate at the Township Level in Taiwan, in 1990 and 2000. International Real Estate Review 10 (1), pp:119-150.

Huang, C., Chen, Y., Wu, J., Li, L and Liu, R. 2015. An evaluation of Suomi NPP-VIIRS data for surface water detection. Remote Sensing Letters 6 (2), pp:155-164.

Jing, X., Shao, X., Cao, C., Fu, X and Yan, L. 2015. Comparison between the Suomi-NPP Day-Night Band and DMSP-OLS for Correlating Socio-Economic Variables at the Provincial Level in China. Remote Sensing 8 (1), pp:17.

Letu, H., Hara, M., Tana, G and Nishio, F. 2012. A Saturated Light Correction Method for DMSP/OLS Nighttime Satellite Imagery. IEEE Transactions on Geoscience \& Remote Sensing 50 (2), pp:389-396.

Li, X., Xu, H., Chen, X and Li, C. 2013. Potential of NPP-VIIRS Nighttime Light Imagery for Modeling the Regional Economy of China. Remote Sensing 5 (6), pp:3057-3081.

Liu, Z., He, C., Zhang, Q., Huang, Q and Yang, Y. 2012. Extracting the dynamics of urban expansion in China using DMSP-OLS nighttime light data from 1992 to 2008. Landscape \& Urban Planning 106 (1), pp:62-72.

Ma, L., Wu, J., Li, W., Peng, J and Liu, H. 2014. Evaluating Saturation Correction Methods for DMSP/OLS Nighttime Light Data: A Case Study from China's Cities. Remote Sensing 6 (10), pp:9853-9872.

Miller, S. D., Mills, S., Elvidge, C. D., Lindsey, D. T., Lee, T. F and Hawkins, J. D. 2012. Suomi satellite brings to light a unique frontier of nighttime environmental sensing capabilities. Proceedings of the National Academy of Sciences of the United States of America 109 (39), pp:15706-15711.

Miller, S. D., Straka, W., Mills, S. P., Elvidge, C. D., Lee, T. F., Solbrig, J., Walther, A., Heidinger, A. K and Weiss, S. C. 2013. Illuminating the Capabilities of the Suomi National PolarOrbiting Partnership (NPP) Visible Infrared Imaging Radiometer Suite (VIIRS) Day/Night Band. Remote Sensing 5 (12), pp:67176766.

Ou, J., Liu, X., Li, X., Li, M and Li, W. 2015. Evaluation of NPPVIIRS Nighttime Light Data for Mapping Global Fossil Fuel Combustion CO2 Emissions: A Comparison with DMSP-OLS Nighttime Light Data. Plos One 10 (9):e0138310.

Powell, B. 2010. Ghost City. Time.

Qingdao Statistics Bureau, 2017. Statistics Communique Qingdao's Economic And Social Development In the Year Of 2016.
Rating and Valuation Department of the Government of Hong Kong Special Administrative Region of the People's Republic of China, 2011 Hong Kong property Review.

Shi, K., Huang, C., Yu, B., Yin, B., Huang, Y and Wu, J. 2014a. Evaluation of NPP-VIIRS night-time light composite data for extracting built-up urban areas. Remote Sensing Letters 5 (4), pp:358-366.

Shi, K., Yu, B., Huang, Y., Hu, Y., Yin, B., Chen, Z., Chen, L and $\mathrm{Wu}$, J. 2014b. Evaluating the Ability of NPP-VIIRS Nighttime Light Data to Estimate the Gross Domestic Product and the Electric Power Consumption of China at Multiple Scales: A Comparison with DMSP-OLS Data. Remote Sensing 6 (2), pp:1705-1724.

Sutton, P., Roberts, D., Elvidge, C and Melj, H. 1997. A Comparison of Nighttime Satellite Imagery and Population Density for the Continental United States. Photogrammetric Engineering \& Remote Sensing 63 (11), pp:1303-1313.

Tang, L., Cheng, H and Qu, G. 2013. Estimating Provincial Economic Development Level of China Using DMSP/OLS Nighttime Light Satellite Imagery. Advanced Materials Research 807-809, pp:1903-1908.

The First National Geographic Census Leading Group Office of the State Council.2017. The Contents and Standards for Basic Geographic National Conditions Monitoring (GQJC 03-2017).

Xie, Y., and Weng, Q. 2016. World energy consumption pattern as revealed by DMSP-OLS nighttime light imagery. Mapping Sciences \& Remote Sensing 53 (2), pp:265-282.

Yang, J. R., and Sun, B. Y. 2004. Development Path for Policy Factors and the Real Estate Market in China _-An Analysis with Game Theory on Government, Developer and Customer. The Study of Finance \& Economics. 30 (4), pp:130-139

Yao, Y., and Li, Y. 2011. House vacancy at urban areas in China with nocturnal light data of DMSP-OLS. IEEE International Conference on Spatial Data Mining and Geographical Knowledge Services.(ICSDM2011), pp:457-462

Zhang, Q., and Seto, K. C. 2013. Can Night-Time Light Data Identify Typologies of Urbanization? A Global Assessment of Successes and Failures. Remote Sensing 5 (7), pp:3476-3494.

Zhang, X., Wu, J., Peng, J and Cao, Q. 2017. The Uncertainty of Nighttime Light Data in Estimating Carbon Dioxide Emissions in China: A Comparison between DMSP-OLS and NPP-VIIRS. Remote Sensing 9 (8), pp:797.

Zheng, Q., Zeng, Y., Deng, J., Wang, K., Jiang, R and Ye, Z. 2017. "Ghost cities" identification using multi-source remote sensing datasets: A case study in Yangtze River Delta. Applied Geography 80, pp:112-121.

Zhou, Y., Smith, S. J., Elvidge, C. D., Zhao, K., Thomson, A and Imhoff, M. 2014. A cluster-based method to map urban area from DMSP/OLS nightlights. Remote Sensing of Environment 147 (18), pp:173-185.

Zhu, J., and Yan Y. 2011. Study on the Characteristics of Housing Vacancy - Case Study of the Housing Vacancy Rate in Hangzhou. Bulletin of Science \& Technology. 27(1), pp:142-147. 
The International Archives of the Photogrammetry, Remote Sensing and Spatial Information Sciences, Volume XLII-3, 2018 ISPRS TC III Mid-term Symposium "Developments, Technologies and Applications in Remote Sensing", 7-10 May, Beijing, China

Zhuo, L., Ichinose, T., Zheng, J., Chen, J., Shi, P. J and Li, X. 2009. Modelling the population density of China at the pixel level based on DMSP/OLS non-radiance-calibrated night-time light images. International Journal of Remote Sensing 30 (4), pp:1003-1018. 